Revue d'histoire de l'Amérique française

DAO REVUE D.HISTOIRE DE L'AMÉRIQUE FRANÇAISE

\title{
Étude bibliographique sur les Rapports de l'Association de la Propagation de la Foi à Montréal
}

\section{Robert Valois}

Volume 4, numéro 4, mars 1951

URI : https://id.erudit.org/iderudit/801671ar

DOI : https://doi.org/10.7202/801671ar

Aller au sommaire du numéro

Éditeur(s)

Institut d'histoire de l'Amérique française

ISSN

0035-2357 (imprimé)

1492-1383 (numérique)

Découvrir la revue

Citer cet article

Valois, R. (1951). Étude bibliographique sur les Rapports de l'Association de la Propagation de la Foi à Montréal. Revue d'histoire de l'Amérique française, 4(4), 560-567. https://doi.org/10.7202/801671ar d'utilisation que vous pouvez consulter en ligne. 


\section{ETUDE BIBLIOGRAPHIQUE SUR LES RAPPORTS DE L'ASSOCIATION DE LA PROPAGATION DE LA FOI A MONTRÉAL}

L'idée missionnaire qui a présidé à la fondation de Montréal au XVIIe siècle, prolongée tout au long du XVIIIe, n'a jamais quitté l'esprit des chefs spirituels du diocèse, depuis sa fondation en 1836.

Le premier évêque de Montréal, Mgr Jean-Jacques Lartigue n'a-t-il pas écrit en l'un de ses Mandements:

Malgré des difficultés nombreuses, [...] Nous avons envoyé depuis le commencement de notre Episcopat, plusieurs Missionnaires en diverses parties de ce Diocèse [ ... ] et Nous sommes résolus à continuer, chaque année, cette œuvre de miséricorde, si la Providence divine veut bien nous fournir pour cela des ressources suffisantes ${ }^{1}$.

Pour se procurer des "ressources suffisantes", Mgr Lartigue adressa au Souverain Pontife, le 9 octobre 1837, une requête demandant le pouvoir d'ériger en son diocèse l'Association de la Propagation de la Foi, telle que Pauline-Marie Jaricot l'avait instituée à Lyon, en France, le 3 mai 1822, et telle que l'avait approuvée le Pape Pie VII, le 15 mars 1823.

Un Rescrit du Pape Grégoire XVI autorisa, le 7 janvier 1838, le premier évêque de Montréal à réaliser son dessein apostolique. Aussi, le 18 avril suivant, dans le Mandement déjà cité, adressé au Clergé et aux Fidèles de son diocèse, Mgr Lartigue écrivait:

[ ... ] Nous avons établi et établissons par les présentes, dans le Diocèse de Montréal, en Canada, l'Association pour l'Oeu-

1. "Mandement" de Jean-Jacques Lartigue, premier évêque de Montréal, 18 avril 1838, Mandements, Lettres Pastorales, Circulaires et autres documents publies dans le diocèse de Montreal depuis son érection (8 vol., Montréal, 1869-1887), I: 31-37. 
vre de la Propagation de la Foi, avec tous les privilèges, Indulgences et autres avantages, accordés à cette société par les Souverains Pontifes².

L'organisation de l'œuvre montréalaise ne tarda pas. Le 4 juin suivant, Mgr Lartigue adressait aux curés du diocèse une Circulaire annonçant la composition du Conseil diocésain; ce Conseil comprenait: l'Hon. Jules Quesnel, président; M. Paul-Joseph Lacroix, Écuyer, vice-président; M. Olivier Berthelot, Écuyer, trésorier; M. Bernard Leprohon, Écuyer, secrétaire, tous de Montréal; M. Alexis Sauvageau, Écuyer, de Laprairie; M. Joseph Larocque, Écuyer, de Longueuil; M. Joseph de la Broquerie, Êcuyer, de Boucherville, le Docteur Louis-Joseph-Guillaume Cazeneuve, de l'Assomption, et Messire Joseph Quiblier, Vicaire général et Supérieur du Séminaire ${ }^{3}$.

Le travail accompli par ce Conseil vint répondre aux légitimes espoirs de l'Évêque et pour donner à l'œuvre un essor encore plus grand, Mgr Lartigue publiait, le 12 mars 1839, un Mandement dans lequel il revenait sur l'importance du nouvel organisme. Il disait:

Nous vous recommandons de nouveau la société inappréciable de la Propagation de la Foi chez les Nations sauvages et dans les autres Missions de ce diocèse, telle que Nous l'établîmes l'année dernière, comme une source de salut $[\ldots]^{4}$

Et comme preuve que ce travail était digne d'encouragement et méritait les honneurs de la publicité, paraissait, à la fin de la première année d'existence de l'Association, le premier numéro d'une revue, destinée à en promouvoir les intérêts, le Rapport de l'Association de la Propagation de la Foi.

On pouvait lire dans l'Avant-Propos:

[... ] On publie en Europe les Annales de la Propagation de la $F o i$, où les vrais Fidèles voient avec une merveilleuse édification les triomphes de leur sainte religion, et les fruits admirables que produisent les Missions. C'est dans ces Annales

2. Ibid., 36.

3. "Circulaire", 4 juin 1838, Ibid., 42.

4. "Mandement", 12 mars 1839, ibid., 49. 
qu'ils vont puiser cette ardeur qui les porte à participer, autant qu'il est en eux, à une ceuvre aussi excellente [...]

Nous croyons done intéresser vivement les bons Catholiques de ce Diocèse, en offrant à leur piété le présent Rapport, qui les mettra au courant des ouvres de la divine miséricorde qui s'opèrent près d'eux et par eux. En lisant ce que le Seigneur a daigné faire en faveur des Domestiques de la Foi, et des Infidèles, qui ont eu le bonheur d'entrer dans le sein de l'Eglise, chacun des membres attachés à l'Association pour la Propagation de la Foi, établie ici l'an dernier, pourra bénir le Père des lumières, de qui vient tout don parfait, de l'avoir associé à une œuvre si grande qu'elle a fait l'objet de la Mission d'un Dieu sur la terre ${ }^{5}$.

Ainsi se présentait le premier numéro d'une série de Rapports qui devait en compter vingt-neuf, répartis sur une période de trentequatre ans. Nous avons sous les yeux cette précieuse collection que conserve jalousement la bibliothèque du Séminaire de Joliette; pour être complète, la collection recevrait avec reconnaissance le Rapport de janvier 1850, dont nous n'avons qu'une copie du sommaire.

Les trois premiers numéros mentionnent sur la couverture les dates suivantes: Mai, 1839. - No. 1; Juin, 1840. - No. 2; Juillet, 1841. - No. 3; Nous avons identifié le quatrième numéro, qui n'a plus de couverture ni de page-titre, par le rapport financier qui, à la page 82, mentionne les dates ultimes de l'État des Dépenses de l'Association, Depuis le 1er juillet 1831, au 1er Décembre 1842, ce qui donnerait comme date probable: Décembre, 1842. - No. 4.

Pour comprendre maintenant que le cinquième Rapport portera - sans numéro d'ordre - une date aussi éloignée que Janvier 1850, il faut un peu revenir en arrière. Peu de temps après que parut le premier Rapport, la Sacrée Congrégation de la Propagande, par une lettre de son Préfet, le Cardinal Fransoni, en date du 16 novembre 1839, invitait Mgr Lartigue à affilier son œuvre diocésaine de la Propagation de la Foi "à l'Association de même nature qui a son Bureau Central à Lyon"'.

Quelle est la raison qui a fait écrire l'évêque de Montréal à Mgr Signay, évêque de Québec, le 6 janvier 1840, pour lui dire

5. Rapport de l'Association de la Propagation de la Foi, I (mai 1839): 3 et 4.

6. "Lettre du Cardinal Fransoni à Mgr de Montréal", Rome, 16 novembre 1839 , Rapport de l'Association de la Propagation de la Foi, [4' (décembre 1842 (?) ], 69. 
qu" il ne répondra pas au Cardinal Préfet de la Propagande qui l'invite à unir la Propagation de la Foi de Montréal à celle de Lyon, en France"" ? Quel est le motif qui lui fit répéter de nouveau dans une lettre au même, le 30 janvier suivant, qu"'il regarde toujours comme non avenue la lettre du Préfet de la Propagande, invitant l'Oeuvre de la Propagation de la Foi de Montréal à s'unir à celle de Lyon"8? Ce sera à Mgr Ignace Bourget de solutionner le problème, après la mort de Mgr Lartigue, survenue le 19 avril 1840.

Le deuxième évêque de Montréal, apôtre missionnaire comme pas un, absolument en faveur de l'indépendance de nos propres missions, fit valoir comme raisons de l'hésitation montréalaise à donner suite au projet, l'opposition du gouvernement anglais, et la défiance qu'inspirerait aux associés canadiens la dissolution des Conseils de Montréal et de Québec ${ }^{9}$. Le 12 novembre 1841, le baron de Jessé, président du Conseil Central de Lyon, dans une longue lettre a Mgr Bourget, donnait des explications satisfaisantes qui dissipèrent toute hésitation. Dans une séance du 29 avril 1842, les membres du Conseil de Montréal signaient la résolution suivante:

[ ... ] Conformément aux intentions de N.S. P. le Pape Gr6goire XVI, aux désirs de Mgr l'évêque de Montréal et à celui du Conseil Général de l'association pour la Propagation de la Foi, établi à Lyon, vû les avantages considérables qui doivent en revenir à l'association de ce diocèse:

Résolu à l'unanimité que cette dernière soit et est de ce moment unie à l'Association générale pour ne plus former qu'une seule et même association et qu'une lettre soit écrite dans ce sens et pour cette fin par les membres de ce diocèse au Conseil Général à Lyon ${ }^{10}$.

Mgr Bourget écrivit donc, le 2 mai suivant, la "lettre" proposée et l'envoya à Lyon avec copie de la résolution de son Conseil. L'union des deux cuvres était scellée.

7. "Correspondance [ analysée] de Mgr Lartigue", lettre du 6 janvier 1840 Rapport de l'Archiviste de la Province de Québec pour 1945-1946 (Redempti Paradis, Imprimeur du Roi, 1946), 124.

8. Ibid., 126.

9. Rapport de l'Association de la Propagation de la Foi, [4 (décembre 1842 (?)], 71.

10. Ibid., 79. 
Voilà ce qui explique qu'après 1842 , les Rapports de l'Association de la Propagation de la Foi ont cessé de paraitre à Montréal. Jusqu'à la reprise de la publication des Rapponts montréalais, les Annales de la Propagation de la Foi, publiées à Lyon, devinrent, pour le diocèse de Montréal, l'organe officiel de toutes les œuvres missionnaires affiliées. Dans ces Annales, qui paraissaient six fois par année, on peut retracer quelque peu l'histoire de nos missions canadiennes, en consultant les Volumes XIV à XXI, des Numéros 80 à 127, années 1842 à 1849.

Nouvel ordre de choses, la répartition des aumônes recueillies dans le diocèse de Montréal, destinées jusqu'alors aux missions dépendant de l'Ordinaire, relevait, par le fait de l'affiliation, du Conseil Général de Lyon.

On eut bientôt à se plaindre du point de vue qui guidait le Conseil général. Une année, Lyon demanda que toutes les aumônes du diocèse de Montréal fussent versées dans les mains de l'Évêque de Buffalo ${ }^{11}$. Les choses ne pouvaient en rester là. Mgr Bourget, dans une Lettre pastorale, adressée, le 19 mars 1851, au Clergé, aux Communautes et aux Fidèles de son Diocèse pour l'encouragement des Associations de la Propagation de la Foi et de la Colonisation, disait:

[... ] Nous allons rétablir le Conseil central de Montréal, que notre union avec l'Association de Lyon nous avait engagé à supprimer. Mais nous aurons, pour nous justifier de ce rétablissement auprès de ce Bureau, des raisons péremptoires. Les voici en deux mots.

Nous avons à fixer, sur notre sol, les domestiques de la foi, nos propres concitoyens. Un des moyens les plus efficaces est de faire marcher à la tête des colons le Prêtre, la croix à la main. Il faut à ce peuple colonisateur des temples et des Ministres du Seigneur. Mais il est pauvre; et dans les commencements qui en fera les frais? L'Association de la Propagation de la Foi, si l'appel que nous faisons aujourd'hui est entendu' ${ }^{12}$.

11. "[ Petite notice sur ] L'Oeuvre de la Propagation de la Foi", Annales de la Propagation de la Foi pour le diocèse de Montréal, 29 (1er juin 1874), 4 de la couverture.

12. "Lettre pastorale" de Ignace Bourget, évêque de Montréal, 19 mars 1851, Rapport de l'Association de la Propagation de la Foi, [6 ] (janvier 1851), 14. 
De là à reprendre la publication de nos Rapports, il n'y avait qu'un pas. Le Rapport, le cinquième de la série, porte la date de Janvier 1850, sans numéro d'ordre, et d'après la lecture du sommaire, il ne semble pas qu'on y ait glissé un mot d'explication sur les raisons de sa réapparition. Il faudra attendre le Rapport de Janvier 1851, pour y trouver la lettre, ci-haut citee, de Mgr Bourget:

Sera la Présente Lettre Pastorale insérée dans le Numéro des Annales de l'Association de la Propagation de la Foi, de la présente année ${ }^{13}$.

Ce même Rapport de Janvier 1851 jette encore un peu de lumière sur les raisons qui ont fait reprendre la publication diocésaine de Montréal. A la page 18, on donne un Coup d' ail sur toutes les Missions du Monde. En préambule à ce Coup d'œil, on écrit:

En présentant ce rapport des missions canadiennes aux pieux Associés de la Propagation de la Foi, notre intention est de pouvoir nous étendre plus au long sur des missions qui nous environnent, et sur celles qui, quoiqu'éloignées de nous, ont été fondées par le zèle de nos compatriotes, comme celle de l'Orégon. Les matières étant trop abondantes pour les Annales publiées en France, nos missions ne peuvent y obtenir qu'un bien petit espace; cependant ce sont celles qui doivent nous intéresser le plus, c'est pour cela que nous publions ce numéro supplémentaire [... ] Cependant avant d'en venir à nos missions, il nous paraît opportun de donner un aperçu des autres missions, ou au moins de celles dont il est parlé dans les Annales de l'année qui se termine. C'est ce que nous allons faire en quelques mots ${ }^{14}$.

Mgr Bourget attachait à ces publications une importance tout apostolique. La même lettre du 19 mars 1851 dit à ce sujet:

Lisez, N.T.C.F., avec attention, et attachez-vous à bien comprendre les Annales de la Propagation de la Foi [ ... ] Conservez soigneusement ces Annales, qui diront à vos enfans, et petits enfans ce que faisaient leurs pères, pour la gloire de la Religion, l'honneur de la Paroisse, le bonheur de la famille ${ }^{15}$.

13. Ibid., 15.

14. Ibid., 18.

15. Ibid., 12 et 13. 
Voici, en terminant, la liste des Rapports de l'Association de la Propagation de la Foi pour le District de Montréal, parus après [ le sixième ], Janvier 1851:

Janvier 1852

Avril 1853

Pour les années 1859-54

Pour l'année 1859

Pour l'année 1860

N.B. A partir de ce numéro le mot Diocèse remplace le mot District dans le titre.

11e Numéro [ sic ] Pour l'année 1861

12 e Numéro [ sic ] Pour l'année 1862 et 1863

$13 e$ Numéro [ sic ] Pour les années 1864 et 1865

$13 e$ Numéro [ sic ] Pour l'année 1869

14 e Numéro [ sic ] Pour l'année 1870

Quinzième Numéro [ sic ] Janivier 1871

Seizième Numéro [ sic ] Mai 1871

Dix-septième Numéro [ sic ] Septembre 1871

Dix-Huitième Numéro [ sic ] Janvier 1872

Dix-Neuvième Numéro [ sic ] Février 1872

Vingtième Numéro [ sic ] Avril 1872

Vingt-Unième Numéro [ sic ] Juin 1872

Vingt-Deuxième Numéro [ sic ] Août 1872

Vingt-Troisième Numéro [ sic ] Octobre 1872

Vingt-Quatrième Numéro [ sic ] Décembre 1872

Vingt-Cinquième Numéro [ sic ] Février 1873

Vingt-Sixième Numéro [ sic ] Juin 1878

Vingt-Septième Numéro [ sic ] Septembre 1873

Bien que le dernier Rapport porte le numéro vingt-sept, on constate que vingt-neuf ont paru. L'erreur de numérotation apparaît pour une part, en ce fait que, parmi les Rapports publiés entre 1850 et 1860, sans numéro d'ordre, il y en a un qui semble avoir échappé au responsable de la numérotation, et, d'autre part, le numéro 13 a été doublé.

Pour le bénéfice des bibliographes, nous ajoutons ici les détails suivants sur la continuation jusqu'à nos jours de la publication de l'Association de la Propagation de la Foi. 
Après le dernier Rapport, paru en septembre 1873, sous le numéro vingt-sept, la revue continue à paraître sous le titre d' $A n$ nales de la Propagation de la Foi por.r le diocèse de Montréal, continuant aussi la même numérotation; ainsi, le numéro publié après le dernier Rapport porte la mention: Vingt-Huitième Numéro. - 1er Février 1874; cette numérotation se continuera encore jusqu'au Quarante-Troisième Numéro, Décembre 1876.

Ici, à la suite d'une entente entre les Conseils de Montréal et de Québec, la revue s'intitule: Annales de la Propagation de la Foi pour la Province de Québec et l'on commence une nouvelle numérotation; ce qui donne comme suite immédiate au Quarante-Troisième Numéro de l'ancienne série, le Premier Numéro, Février 1877, de la nouvelle série. Au Trentième Numéro, Octobre 1886, de cette série, le titre subit encore une modification: désormais on aura les Annales de la Propagation de la Foi pour les Provinces de Québec et de Montréal; cette série se continuera jusqu'au Cent Quarante et U'nième Numéro, Octobre 1923.

Le Motu Proprio du Pape Pie XI, Romanorum Pontificum, du 3 mai 1922, ayant fusionné toutes les sociétés particulières de la Propagation de la Foi en une société universelle, dont le siège est fixé à Rome, il y eut une réorganisation dans tous les pays du monde où l'Association existait. Au Canada deux Conseils Nationaux furent formés, l'un pour l'Est, avec siège à Québec, l'autre pour l'Ouest, avec siège à Toronto. Le Conseil National du Canada oriental, érigé par Rome, le 18 décembre 1922, s'occupa toute l'année 1923 à réorganiser l'œuvre, et le 10 janvier 1924, Rome en approuvait les Statuts ${ }^{16}$. Le numéro 23 de ces Statuts se rapporte à la publication des Annales. En Février 1924, paraissaient les Annales de la Propagation de la Foi, Nouvelle série, Edition française, Vol. 1, No 1. C'est la série qui paraît encore.

Robert VALOIS, c.s.v. Seminaire de Joliette (Québec)

16. Annales de la Propagation de la Foi, Nouvelle série (Edition française publiée au Canada), I (février 1924): 9. 